\title{
AN INTERACTIVE LOGISTICS CENTRE INFORMATION INTEGRATION SYSTEM USING VIRTUAL REALITY
}

\author{
S. Hong ${ }^{1}$, B. Mao ${ }^{1, *}$ \\ College of Information Engineering, Collaborative Innovation Centre for Modern Grain Circulation and Safety, Jiangsu Key \\ Laboratory of Grain Big-data Mining, Nanjing University of Finance \& Economics, Nanjing, 210023, China - hjmrezl@ outlook.com, \\ maoboo@gmail.com
}

Commission III Urban Sensing and Mobility

KEY WORDS: Logistics Centre, Virtual Reality, Video Surveillance, Eye Tracking, Logistics Traceability, Semantically Labelled Data

\begin{abstract}
:
The logistics industry plays a very important role in the operation of modern cities. Meanwhile, the development of logistics industry has derived various problems that are urgent to be solved, such as the safety of logistics products. This paper combines the study of logistics industry traceability and logistics centre environment safety supervision with virtual reality technology, creates an interactive logistics centre information integration system. The proposed system utilizes the immerse characteristic of virtual reality, to simulate the real logistics centre scene distinctly, which can make operation staff conduct safety supervision training at any time without regional restrictions. On the one hand, a large number of sensor data can be used to simulate a variety of disaster emergency situations. On the other hand, collecting personnel operation data, to analyse the improper operation, which can improve the training efficiency greatly.
\end{abstract}

\section{INTRODUCTION}

The rapid development of information technology in modern society has accelerated the tendency of e-commerce to lead the economic transformation and development (Sila, 2013; Bask et al. 2012). Logistics is one of the most important factors in realizing and sustainable development of e-commerce (Yu et al. 2016). It is playing an increasingly important role in modern city operations. The development of the logistics industry produces various information such as video monitoring, environment sensing and accurate positioning to effectively monitor, and configure logistics resources (Kong et al. 2017). Therefore, more and more surveillance devices are equipped in the logistics centre to monitor temperature, humidity, video information, number of inventory and etc. in real time.

To integrate the various data for better understanding, this paper combines with virtual reality technology which can simulate real scene very vividly, create a VR based system to visualize and interact the monitoring information from the logistics centre. In this proposed system, environment information (temperature, humidity, $\mathrm{CO} 2$ and others) is visualized in the VR platform by volume rendering with visual variable mapping algorithms. The video surveillance data is analysed using object detection, then the detected objects such as people, vehicles or goods are modelled and visualized in the VR environment. Furthermore, when a user checks the logistics centre data with our VR platform, we record all the interaction of the user with eye tracking equipment.

The rest of this paper is structured as follows: in Section 2, we described some related works of our study. In Section 3, we

\footnotetext{
* Corresponding author

National Key Technologies Research and Development Program of China under Grant 2015BAD18B02
}

interpreted our system framework and give user's data acquisition, which is implemented in Section 4. Finally, Section 5 concludes and analyses the study.

\section{RELATED WORK}

\subsection{Logistic Traceability}

With the awakening of people's safety awareness, the demand for product safety is increasing, and the transparency of product information has become the mainstream of current market development. The application of traceability technology in product safety is essential. The traceability technology has been applied successfully in various food safety supply chains and has played an irreplaceable role (Xu et al. 2006). The system proposed by this paper is based on the logistics traceability. In logistics, traceability refers to the capability for tracing goods along the distribution chain on a batch number or series number basis. Traceability is an important aspect for example in the automotive industry, where it makes recalls possible, or in the food industry where it contributes to food safety (Li et al. 2007).

The international standards organization EPCglobal under GS1 has ratified the EPCglobal Network standards (especially the EPC Information Services EPCIS standard) which codify the syntax and semantics for supply chain events and the secure method for selectively sharing supply chain events with trading partners (Qiao et al. 2007). These standards for traceability have been used in successful deployments in many industries and there is now a wide range of products that are certified as being compatible with these standards. 


\subsection{Virtual Reality}

Virtual reality, is a high-level computer interface based on immersion, interactivity and conception, the comprehensive utilization of computer graphics, simulation technology, multimedia technology, artificial intelligence technology, computer network technology, parallel processing technology and sensor technology, simulating human visual, auditory, tactile and other sensory organ functions, that can make people immersed in a virtual scene generated by computer, interact with it in real time by language, gestures and other natural ways, which creates a human-oriented multi-dimensional information space.

Virtual reality technology has virtuality beyond reality, and the core equipment of the system still is the computer. Image display devices are the key peripherals used to create the stereoscopic visual effect, commonly used are 3D projectors and helmet displays etc. Currently, frequently used helmet displays are Oculus Rift, HTC Vive, and SONY PlayStation VR. The above three virtual reality helmets are integrated head tracking, position tracking system, there is little difference in hardware with only minor differences between them. Oculus Rift and HTC Vive are equipped with a monocular resolution of $1080 * 1200$ pixels OLED display, the visual angle of 110 degrees, the refresh rate of $90 \mathrm{~Hz}$, but the Oculus Rift screen ratio of 16: 9, HTC Vive is 9: 5. Relatively speaking, the Sony PlayStation VR has a slightly lower screen resolution of $960 *$ 1080 pixels and visual angle of 100 degrees, but the refresh rate reaches up to $120 \mathrm{~Hz}$. This paper uses the HTC Vive helmet.

Virtual environment represents a controllable alternative to field studies conducted in the real world. (Bertrand et al. 2013) conducted a path-finding study based on the virtual reality system. (Helmut et al. 2016) combined the virtual reality environment with a mobile eye tracking device, proposed a novel navigation evaluation system. There is no research on the grain logistics centre in the virtual reality environment at present. In this study, we combine VR helmet with eye tracking module in virtual reality environment to realize an interactive logistics centre information integration system, which greatly reduces the research cost and enhances the significance of research on tracing the source of logistics in VR.

\section{SYSTEM FRAMEWORK}

We implement a basic test platform for a grain logistics centre, as shown in Figure 1.

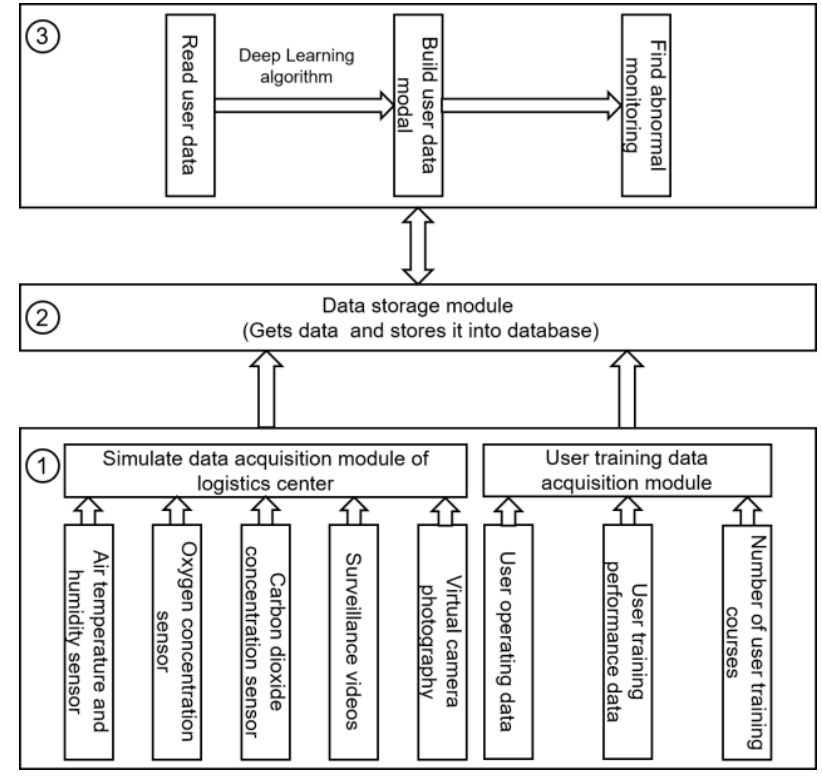

Figure 1. VR Based Logistic Centre Information Integration System

\subsection{Framework Interpretation}

From above system framework of this paper, we can conclude that the whole study mainly divided into three parts. Firstly, we simulate to acquire the grain logistics centre various sensors data in the scene, such as virtual camera photography, surveillance videos, the carbon dioxide concentration sensor, oxygen concentration sensor, air temperature and humidity sensor. Meanwhile, user's behaviour data such as User training data, User operating data can also be obtained by HTC Vive, and Eye Tracking Module. The data obtained above will be stored in a relational database. At last, we use deep learning method to analyse the data, by reading the data from relational database, then use deep learning algorithm to train the data and build two predict models, one of the models can be used to predict user's future action, it's convenient for executives customizing the training program for users, another model is used to find the grain logistics centre abnormal monitoring.

\subsection{Data Collection}

There are many ways to get points in 3D world coordinates system. (Munn et al. 2008) proposed a method where the 3D Point of Regard (POR) is estimated from a portable monocular video-based eye tracker applying computer vision techniques to get 3D structure and motion from video sequences. (Pirri et al. 2011) proposed a model-based approach for $3 \mathrm{D}$ gaze estimation for wearable multicamera devices. The methods described above are relatively complicated. In this paper, we get user's gaze points by the collision points between Steam VR ray and these observed objects, this method is simple and can guarantee the success rate of data acquisition. The obtained data is written to disk through the C\# file stream, as shown in Figure 2. 


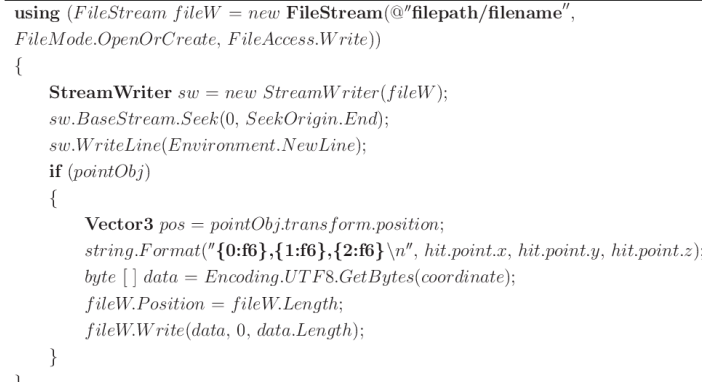

Figure 2. Using C\# stream to write users' data

\section{IMPLEMENTATION OF SYSTEM}

To implement this paper proposed system, in the primary step, this demo system introduces real logistics centre scenes (Figure 3.) into OBJ format model according to the proportion of $1: 1$, and import it into Unity 3D software, then combines with Steam VR technology to display the logistics centre scene in the HTC Vive virtual reality helmet, as shown in Figure 4.

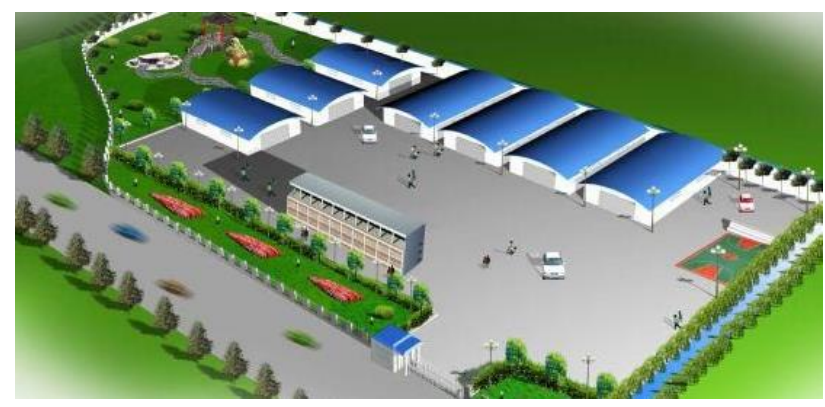

Figure 3. Logistics Centre Scene

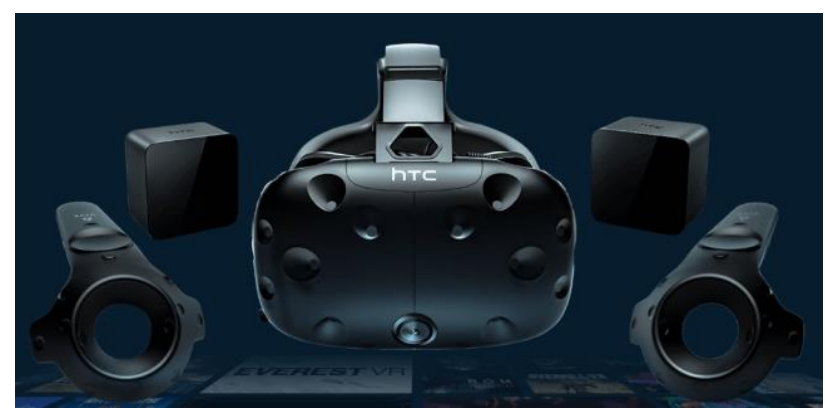

Figure 4. HTC Vive Virtual Reality Helmet

Then, using the Steam VR 1.0 tracking technology and HTC Vive handles to realize panoramic roaming (Zhang et al. 2016). In the scene, we can use the Unity 3D camera to render and simulate the real monitoring function to collect the monitoring data according to the actual situation of the logistics centre (Figure 5.). Meanwhile, using the HTC Vive handle interactive function to simulate the outburst safety misadventure such as a fire accident by reducing the previous safety incidents related to logistics warehousing, thereby, can realize the education and training of the logistics centre managers for safety accidents. This system also can record the personnel's operating data during the training process. Thus, we can find the monitoring anomaly by analysing the acquired user data through a deep learning algorithm, to realize the function of user behaviour analysis, which has a practical significance for the training of warehousing managers in the logistics centre.

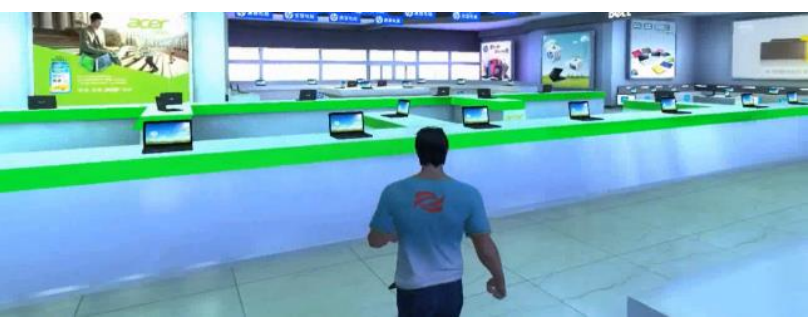

Figure 5. Roaming in Logistics Centre

\section{CONCLUSION AND ANALYSIS}

The proposed VR based framework of this paper has some innovative significance, using the immersion of virtual reality, users can immerse themselves in the real logistics centre scene without leaving the house, and can repeat the training of logistics centre safety supervision, and the importance of framework is that not only surveillance data is integrated and visualized for better understanding, but also the user interactivities are recorded which is essential to generate the semantically labelled data automatically. The semantic labelled information could be further used to train the data mining models such as deep learning network for automatic logistic centre semantic monitoring and alerting system (Rimienè et al. 2007). For example, abnormal detection is important to the safety of logistic centre, but it is difficult to define what is abnormal. With the help of the proposed system, we can monitor the interaction between user and surveillance data from that the objective opinions to a phenomenon in a logistic centre from the users are recorded. These objective opinions including watching time, the way of checking, zoom in/out and etc. will be further classified into normal or abnormal reactions which build a bridge between surveillance data and its semantic meaning automatically.

\section{ACKNOWLEDGEMENTS}

This paper is supported by National Key Technologies Research and Development Program of China under Grant 2015BAD18B02.

\section{REFERENCES}

Sila, I., 2013. Factors affecting the adoption of B2B Ecommerce technologies. Electronic Commerce Research, 13(2), pp. 199-236.

Bask, A., Lipponen, M., and Tinnilä, M., 2012. E-commerce logistics: a literature research review and topics for future research. International Journal of E-Services and Mobile Applications, 4(3), pp. 1-22.

Yu, Y., Wang, X., Zhong, R.Y., and Huang, G.Q., 2016. Ecommerce Logistics in Supply Chain Management: Practice Perspective. Procedia Cirp, 52, pp. 179-185.

Kong, X.T.R., and Luo, H., 2017. Reconfigurable express logistics centre: a simulation study. IFAC-Papers Online, 50(1), pp. 11770-11775. 
Xu, S.W., Li, Z.Q., and Lim, Z., 2006. Study on China Food Safety Information Sharing and Public Management System. China Agriculture Press, Beijing. pp. 31-34.

Li, G.M., Huang, L.P., and Zhan, J.C., 2007. RFID Application in Food Safety Traceability. In: Logistics and Material Handing, Vol. 12, pp. 85-87.

Qiao, J., Han, Y., Li, and D. L., 2007. Importance and Restricting Factor Analysis on Implementing Food Safety Traceability in China. Guide to Chinese Poultry, 43(6), pp. 1012.

Bertrand, J., Ebrahimi, E., Wachter, A., Luo, J., Babu, S. V., and Duchowski, A. T., 2013. Visual attention to wayfinding aids in virtual environments. In: Proceedings of the 5th Joint Virtual Reality Conference, pp. 9-16.

Helmut, S. F., Volker, S., and Stefan, S., 2016. Evaluation of indoor guidance systems using eye tracking in an immersive virtual environment. Spatial Cognition and Computation, 17(12), pp. 163-183.

Munn, M. S., and Pelz, J. B., 2008. 3D point-of-regard, position and head orientation from a portable monocular video-based eye tracker. In: Proceedings of the Symposium on Eye Tracking Research \& Applications, pp. 181-188.

Pirri, F., Pizzoli, M., and Rudi, A., 2011. A general method for the point of regard estimation in 3D space. In: IEEE Conference on Computer Vision and Pattern Recognition. IEEE, Vol. 32, pp. 921-928.

Zhang, K.F., Zhao, X., and Xie, Y.L., 2016. VR and AR develop actual combat. China Machine Press, Beijing. pp. 239- 240.

Rimienè, K., and Grundey, D., 2007. Logistics Centre Concept through Evolution and Definition, Engineering Economics, 31 (4), pp. 9. 